\title{
Guided Wave Optical MEMS Pressure Sensor
}

\author{
Prasant Kumar Pattnaik, A. Selvarajan and T. Srinivas \\ Department of Electrical Communication Engineering \\ Indian Institute of Science, Bangalore 560012, India \\ Telephone: +91-80-2293 2852 Fax:+91-80-2360 0563 \\ Email: pattu@ece.iisc.ernet.in
}

\begin{abstract}
Guided wave Optics in combination with micromachining technology offer immense potential for sensor applications. In this paper we analyse an guided wave optics based MEMS pressure sensor consisting of a Mach-Zehnder interferometer whose sensing arm is over the edge of a bulk micromachined silicon diaphragm. We compare this sensor utilising the elastooptic effect with the conventional piezo-resistive and capactive micromachined pressure sensors in terms of their sensitivities. This MOEM pressure sensor is found to be superior to the conventional ones. This sensor can be used for blood pressure monitoring, precision instrumentation, aerospace propulsion application with suitable design parameters.
\end{abstract}

\section{INTRODUCTION}

Micro-opto-electro-mechanical (MOEM) devices and systems, based on the principles of integrated optics and micromachining technology on silicon have immense potential for sensor applications [?]. Employing optical techniques have important advantages such as functionality, large bandwidth and higher sensitivity. Pressure sensing using micromachined structures have utilized the changes induced in either the resistive or capacitive properties of the electro-mechanical structure by the impressed pressure [?]. Integrated optical pressure sensors can utilize the changes to the amplitude, phase, refractive index profile, optical path length, or polarization of the lightwave by the external pressure. This type of pressure sensor can find application in blood pressure sensor, aerospace propulsion and other harsh enviroments and precision instrumentation with suitable design parameters.

Many opto-mechanical pressure sensors have been reported earlier. In the earliest reported work by Ohkawa et.al [?] novel integrated optic presure sensor was constructed with MachZehnder structure of glass waveguides on silicon substrate. Pressure is sensed by detecting the deformation of thin diaphragm through MZI. Wagner et. al [?] improved this by further integrating pin photodiode along with the same sensor chip. Beniassa et.al [?] have done the similar sensor but with ARROW waveguides. There are many pressure sensors reported based on Fabry-Perot interferometer e.g [?]. But though this type has higher sensitivity, they are limited to low pressure ranges. Here we consider the guided wave MOEM Pressure sensor consisting of a Mach-Zehnder Inteferometer (MZI) whose sensing arm is over the edge of a silicon micromachined diaphragm [?]. As the diaphragm deflects due to the applied pressure, due to the stress, the refractive index of the waveguide changes and hence the phase of the light propagating through it. This phase change is converted to intensity change using the MZI. The approach is similar to Benaissa but we have designed the ARROW for $1.3 \mu \mathrm{m}$ wavelength, so that hybrid integration of the source is also possible with the sensor on the same chip. Also we have modeled and carried out the frequency response of the sensor. We have compared this sensor with other MEMS sensors in terms of their sensitivities.

\section{SENSOR CONFIGURATION}

Figure 1 shows the schematic of the MOEM pressure sensor. The sensing arm of the MZI is over the longer edge of the diaphragm. The MZI is formed by ridge anti-resonant reflecting optical wavegude (ARROW). ARROW consists of stacked layer of silicon dioxide and silicon nitride as shown in the figure 1 . This waveguide has large core size for single-mode, single polarisation behaviour and has low loss.

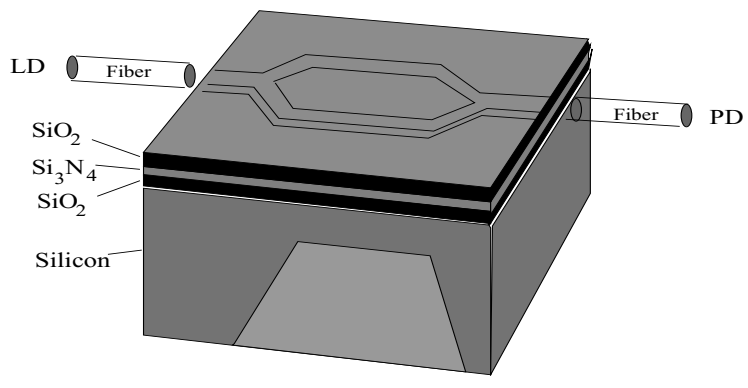

Fig. 1. Schematic of the guided wave optical MEMS Pressure Sensor

\section{DESIGN AND ANALYSIS}

The design of the sensor involves the design of mechanical diaphragm, ridge optical waveguide (ARROW), MZI and the opto-mechanical coupling between.

\section{A. Mechanical Design}

The mechanical design of the diaphragm is carried out using the differential equation

$D \frac{\partial^{4} W}{\partial y^{4}}+2 H \frac{\partial^{4} W}{\partial y^{2} \partial z^{2}}+D \frac{\partial^{4} W}{\partial z^{4}}+c \frac{\partial W}{\partial t}+\rho h \frac{\partial^{2} W}{\partial t^{2}}=P(t)$ 
with boundary condition

$$
\left.W\right|_{z= \pm a / 2, y= \pm b / 2}=0,\left.\frac{\partial W}{\partial z}\right|_{z= \pm a / 2}=0,\left.\frac{\partial W}{\partial y}\right|_{y= \pm b / 2}=0
$$

Where $\mathrm{W}$ is displacement, $D=\frac{E h^{3}}{12\left(1-\nu^{2}\right)}$ is the flexural rigidity and $H=\nu D+2 D_{y z}, D_{y z}=\frac{G h^{3}}{12}, \mathrm{E}$ is Young's modulus, G is shear modulus, $\nu$ is Poisson's ratio, $\rho$ is the density, $\mathrm{h}$ is thickness, $\mathrm{P}$ is the differential pressure, $\mathrm{a}, \mathrm{b}$ and $\mathrm{h}$ are length, width and thickness of the diaphragm and $\mathrm{c}$ is the damping coefficient. The diaphragm is in YZ-plane with origin being its center and $Z$-axis is along its length.

This equation for static pressure is solved using series approximations for the deflection as outlined by Timoshenko [?]. First the displacement $\mathrm{W}$ is obtained and from this different stress components are arrived. The stress component $\sigma_{x x}, \sigma_{x y}$ and $\sigma_{x z}$ are neglected owing to small thickness compared to length and width. Stress components $\sigma_{y z}$ is much smaller compared to $\sigma_{y y}$. Figure 2 shows the stres components $\sigma_{y y}$ and $\sigma_{z z}$ in the normalised form. The normalised stress component $\sigma_{y y}$ is larger than $\sigma_{z z}$ and rectangular diaphragms generate more stress compared to the square diaphragm as evident from the figure 2. Since the MOEM pressure ustilise the photo-elastic
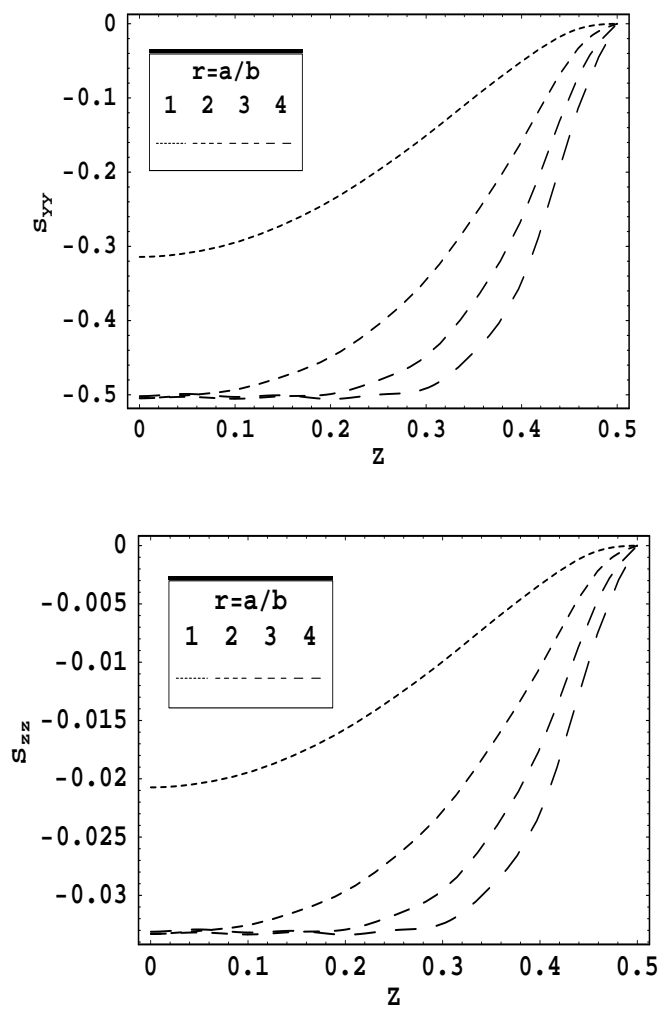

Fig. 2. Normalised stress $\left(P b^{2} / h^{2}\right)$ of (a) $\sigma_{y y}$ and (b) $\sigma_{z} z$ for various aspect ratios effect, more stress generate more index change, rectangular diaphragm is designed. Since longer the length of the diaphragm, more is the accumulated phase, rectangular diaphragm with higher aspect ratio is designed for better response. But for longer dimesnion, frequency response will reduce and hence needs to be carefully designed depending on the application.

The dynamic analysis is carried out as outlined by Soedel [?]. The dimensions of the diaphragm are choosen to meet the dynamic requirements, i.e. frequency response. The normal mode frequency, calculated using the Rayleigh-Ritz approximation taking the beam elements for the diaphragm, is given by

$\omega_{m n}^{2}=\frac{D}{\rho h b^{4}}\left[\left(\frac{\lambda_{m}}{r}\right)^{4}+\lambda_{n}^{4}+2 \frac{H}{D} \frac{\int_{0}^{a} \alpha \frac{\partial^{2} \alpha}{\partial z^{2}} d z \int_{0}^{b} \beta \frac{\partial^{2} \beta}{\partial y^{2}} d y}{\int_{0}^{a} \alpha^{2} d z \int_{0}^{b} \beta^{2} d y}\right]$

where $\alpha$ and $\beta$ are the beam functions and $\lambda_{m}$ and $\lambda_{n}$ are eigen values of the beam function which depends on the length and boundary condition of the beam. From this we obtain

$$
\omega b^{2} \sqrt{\frac{\rho h}{D}}=\text { Constant }
$$

For the fundamental mode $(m=1, n=1)$, we get the constant to be 22.78 for aspect ratio $r \geq 2$. Since longer the length of the diaphragm, more is the accumulated phase, rectangular diaphragm of dimension $4 \mathrm{~mm} \times 1 \mathrm{~mm} \times 20 \mu \mathrm{m}$ is designed for pressure range upto $1 \mathrm{MPa}$. The change in length due to applied pressure is negligible for these dimensions. The fundamental frequency for this diaphragm is found to be $206.5 \mathrm{kHz}$. For better linearity, depending on damping, usable frequency range is determined. For $c=0.1$, the operating frequency range range of $10 \mathrm{kHz}$ can be obtained with a frequency linearity of less than $0.1 \%$ for normalised damping coefficient $\xi=0.7$.

\section{B. Optical Design}

The ARROW waveguide $\left(\mathrm{SiO}_{2} / \mathrm{Si}_{3} \mathrm{~N}_{4} / \mathrm{SiO}_{2}\right)$ is designed for $1310 \mathrm{~nm}(5 / 0.21 / 2.5 \mu \mathrm{m})$ using the transverse resonance method (TRM) [?] and the ridge height and width (1.3 and $5 \mu \mathrm{m})$ are designed using effective index method (EIM). The MZI is designed using the beam propagation method (BPM). The opto-mechanical coupling occurs because of photo-elastic effect. Due to the differential pressure across the diaphragm, stress is generated in it which produces a change in refractive index of the waveguide

$$
\Delta n_{i}=C_{i j} \sigma_{j} \quad i, j=1,2, \ldots 6
$$

where $\mathrm{C}$ is the photo-elastic tensor. Only non-zero coefficients for silicon dioxide are $C_{11}=-0.65 \times 10^{-12} / \mathrm{Pa}, C_{22}=$ $-4.22 \times 10^{-12} / \mathrm{Pa}, C_{44}=C_{11}-C_{22}$. Hence the refractive index change for TE and TM modes are given by

$$
\begin{array}{cc}
\Delta n_{y y}=C_{11} \sigma_{y y}+C_{22} \sigma_{z z} & \text { TE Mode } \\
\Delta n_{x x}=C_{22}\left(\sigma_{y y}+\sigma_{z z}\right) & \text { TM Mode }
\end{array}
$$


Normalised refractive index change (normalised to $\frac{P b^{2} h}{D}$ ) over the whole diaphragm for TM mode is shown in figure 3 and along the longer edge of the diaphragm for bot TE and TM mode is shown in figure 4 for length/width ratio of 4 . Refractive index change is more along the edges and is greater for TM modes than TE modes. This index change pro-

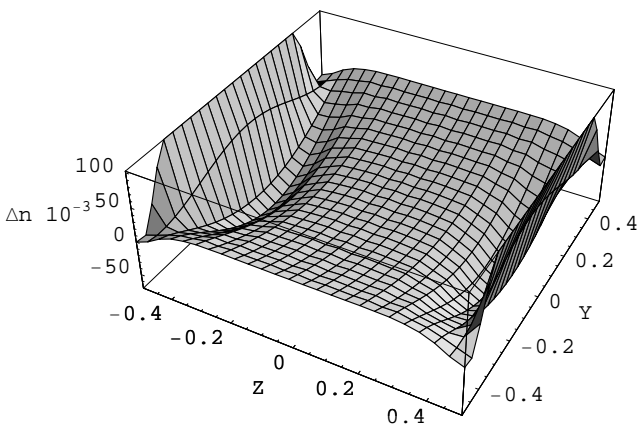

Fig. 3. Normalised change in refractive index over diaphragm for TM mode

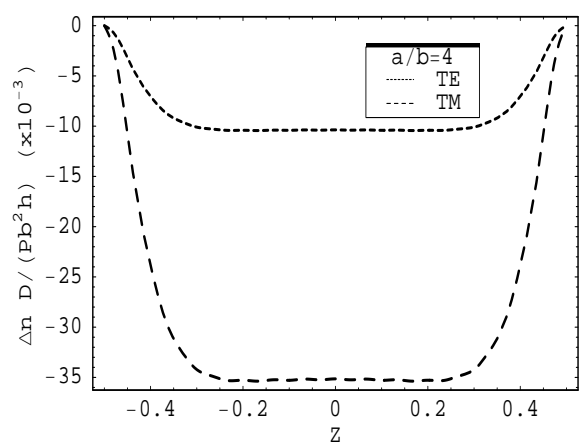

Fig. 4. Normalised change in Refractive Index along the longer edge for TE and TM mode for $\mathrm{a} / \mathrm{b}=4$

duces the phase change in the waveguide. Figure 5 shows the phase change $(\Delta \phi)$ for TE and TM modes for $r=4$ and $b=$ $1 \mathrm{~mm}, h=20 \mu \mathrm{m}$ The phase change is linear with the differential pressure and it is larger for TM polarisation, since the optomechanical coupling $\left(C_{22}\right)$ is larger for the TM mode. The phase sensitivity of the sensor defined as $\frac{\Delta \phi}{P \phi}$ is found to be $90 \mu \mathrm{rad} / \mathrm{Pa}$. Normalised change in output intensity of the interferometer is

$$
\frac{\Delta I}{I}=\operatorname{Sin}^{2}\left(\frac{\Delta \phi}{2}\right)
$$

Figure 6 shows the normalised output intensity change for various applied differential pressure.

The sensitivity of the sensor definded as $\frac{\Delta I}{P I}$ is calculated to be $45.48 \mu \mathrm{W} / \mathrm{mW} / \mathrm{kPa}$ when a detector of quantum efficiency

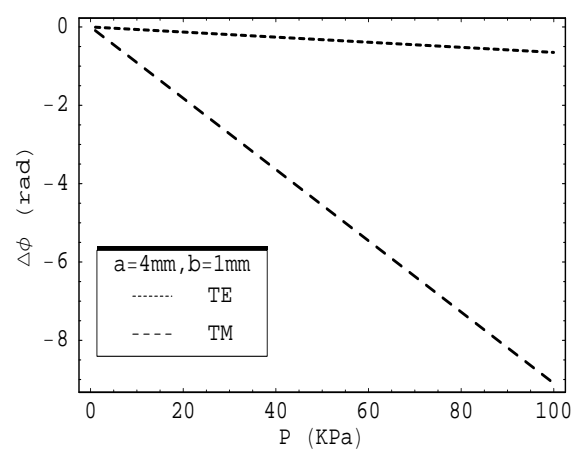

Fig. 5. Phase change for TE and TM modes along the edge vs differential pressure

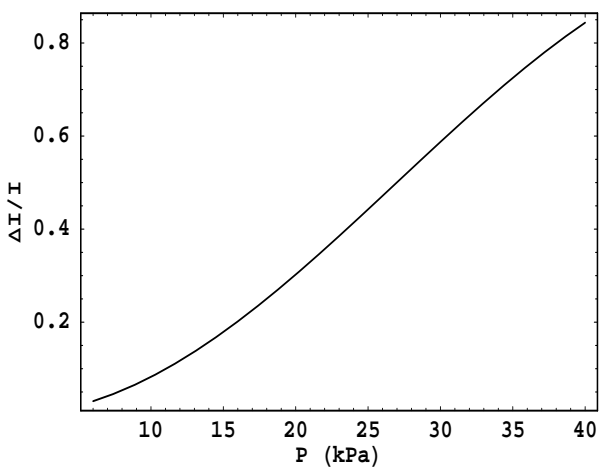

Fig. 6. Normalised change in output vs differential pressure

of 0.8 is used for detection.

\section{COMPARISON WITH PIEZO-RESISTIVE AND CAPACITIVE PRESSURE SENSORS}

The change in resistance due to applied stress for a piezoresistor is given by

$$
\frac{\Delta R}{R}=\pi_{l} \sigma_{l}+\pi_{t} \sigma_{t}
$$

where $\pi_{l}$ and $\pi_{t}$ are longitudinal and transverse piezo-resistive coefficients and $\sigma_{l}$ and $\sigma_{t}$ are the longitudinal and transverse stresses. The stresses at the center of the edge of the diaphragm is maximum and piezo-resistor is placed there. Generally four piezoresistors are kept in a wheatstone bridge configuration for detection. But here we just consider the effect for comparison. For similar diaphragm and for a piezo-resistor of $1 k \Omega$, the sensitivity $\frac{\Delta R}{R P}$ is found to be $8.45 \times 10^{-4} / k P a$. The gauge factor $\frac{\Delta R}{R \epsilon}$ for this is found to be 108 .

The capacitance of the parallel plate capacitor formed by the diaphragm and the ground plane is $C_{0}=\frac{\epsilon A}{d}$, where $\epsilon$ is the dielectric constant of the medium, $\mathrm{A}$ is area of the diaphragm forming the capacitor and $\mathrm{d}$ is the distance between the ground 
plane and the capacitor. Due to the differential pressure, the normalised change in capacitance, for deflection small compared to thickness is given by

$$
\frac{\Delta C}{C}=\frac{1}{d A} \iint W(x, y) d x d y
$$

It is a highly nonlinear function of pressure and hence the pressure range is quite small for good linearity. For the chosen diaphragm and for $d=200 \mu m$, the sensitivity of the capacitive sensor is obtained to be $132.92 \times 10^{-4} / k P a$. Table 1 summarises the comparison of the piezoresistive, capactive and optical pressure sensor.

TABLE I

MEMS AND MOEM PRESSURE SENSOR COMPARISON

\begin{tabular}{|c|c|c|c|}
\hline Sensitivity & Piezo-resistive & Capacitive & MOEM \\
\hline Defination & $\frac{\Delta R}{R P}$ & $\frac{\Delta C}{C P}$ & $\frac{\Delta I}{I P}$ \\
\hline Value & & & \\
$\left(10^{-4} / k P a\right)$ & 8.45 & 132.92 & 454.8 \\
\hline
\end{tabular}

MOEM sensor is seen to be superior in terms of sensitivity. Half wave pressure of the sensor is about $40 \mathrm{kPa}$. The cumulative nature makes it more sensitive. Piezo-resistive sensors are primarily limited by the noise due to variation in temperature. But for optical sensor, noise due to temperature is very small. The capacitive is sensor limited by the mechanicalthermal noise and has smaller dynamic range. The MOEM pressure sensor is primarily limited by the shot noise arising due to detection. For detector of quantum efficiency of 0.8 , the noise equivalent pressure is found to be about $3.83 \times 10^{-5} \mathrm{~Pa}$ which is much smaller than the electronic counterparts.

\section{APPLICATIONS}

The designed sensor has half wave pressure of $40 \mathrm{kPa}$ which is good enough for blood pressure sensing $(300 \mathrm{~mm} \mathrm{Hg})$. Since the lower limit is about $3.83 \times 10^{-5} \mathrm{~Pa}$, it can be used for very low pressure (high vaccum) and precision instrumentation purposes. As the dynamic range is about $10 \mathrm{kHz}$, pressure varying upto this rate can be detected with proper signal processing circuitary. Also due to small temperaturate dependence of refractive index of the core $\mathrm{SiO}_{2}\left(10^{-} 6 /{ }^{0} \mathrm{~K}\right)$, this type of sensors can be used in harsh enviroments such as aerospace application. Multiplexing of the sensors and distributed sensing can be easily done.

\section{CONCLUSION}

MOEM sensing devices have improved characteristics. Here we have analysed and compared MZI based guided wave optical MEMS pressure sensors with piezoresistive and capacitive sensors. Due to cumulative nature of the total phase change (photo-elastic) in the waveguide, this sensor is found to be more sensitive than the electronic counterparts. Also the fundamental noise limit for the guided wave MOEM pressure is much less than the piezo-resistive and capacitive sensors. This type of sensors find apllication in blood presure sensing, precision instrumentation, aerospace and other harsh enviroments with suitable designs.

\section{ACKNOWLEDGEMENTS}

Authors wish to acknowledge National Programme on Smart Materials (NPSM), Govt. of India for supporting the work.

\section{REFERENCES}

[1] M.Tabib Azar and G.Behelm, "Modern trends in microstructures and integrated optics for communication, sensing and signal processing", Opt. Engg., vol.36, no.5, pp. 1307-1318, 1997.

[2] W.P Eaton and J.H Smith, "Micromachined pressure sensors: review and recent developments", Jl. of Smart materials and structures, vol.6, pp.530-539, 1997.

[3] M.Ohkawa, M.Izustu and T.Sueta, "Integrated Optic Pressure Sensor on silicon substrate”, Appl. Opt. , vol. 28, No. 23, pp.5153-5157, 1989.

[4] C. Wagner, J. Frankenberger and P. Deimel, "Optical pressure sensor based on Mach-Zehnder interferometer integrated with a lateral Si:H pi-n photodiode", IEEE Phton. Tech. Lett. vol.5, no. 10, pp.1257-1259, 1993.

[5] K. Benaissa and A. Nathan, "IC compatible opto-mechanical pressure sensor using Mach-Zehnder interferometry", IEEE Trans. Electron Devices, vol.43, no. 9, pp.1571-1581, 1996.

[6] Y. Kim and D.P.Neikrik, "Micromachinded fabry-perot cavity pressure sensor", IEEE Photon. Tech. Lett., vol.7, no.12, pp. 1471-1473, 1995.

[7] T.Srinivas, P.K.Pattnaik, T.Badrinarayana and A.Selvarajan, "Analysis of micro-opto-electro-mechanical (MOEM) Pressue Sensor", Proc. SPIE, Vol.3897, pp.364-371, 1999

[8] S. Timoshenko and S. Woinowsky-Krieger, "Theory of Plates and Shells", McGraw-Hill, NewYork, 1959.

[9] Werner Soedel, "Vibration of Shells and Plates", Marcel Dekker Inc, New York, 1993.

[10] T.Baba and Y. Kokuban, "Dispersion and radiation loss characteristics of antiresonant reflecting optical waveguides- Numerical results and analytical expressions", IEEE J. Quantum Electron., vol.28, pp. 1015-1022, 1992. 